Wolfgang Böhm

Günther Gose

\title{
Einführung in die Methoden der Numerischen Mathematik
}

für Mathematiker, Informatiker und Interessenten der

naturwissenschaftlichen Fächer

Mit zahlreichen Abbildungen

Vieweg 


\section{Dr. Wolfgang Böhm ist Professor am Institut für Angewandte Mathematik der Technischen Universität Braunschweig \\ Dr. Günther Gose ist wissenschaftlicher Mitarbeiter einer großen Versicherungsgesellschaft}

\section{Verlagsredaktion: Alfred Schubert}

CIP-Kurztitelaufnahme der Deutschen Bibliothek

\section{Böhm, Wolfgang}

Einführung in die Methoden der numerischen Mathematik:

für Mathematiker, Informatiker und Interessenten

der naturwissenschaftlichen Fächer / Wolfgang Böhm;

Günther Gose. - 1. Aufl. - Braunschweig: Vieweg, 1977.

ISBN-13: 978-3-528-03029-2 e-ISBN-13: 978-3-322-85528-2

DOI: $10.1007 / 978-3-322-85528-2$

NE: Gose, Günther:

\section{7}

Alle Rechte vorbehalten

(C) Friedr. Vieweg \& Sohn Verlagsgesellschaft mbH, Braunschweig, 1977

Die Vervielfältigung und Übertragung einzelner Textabschnitte, Zeichnungen oder Bilder, auch für Zwecke der Unterrichtsgestaltung, gestattet das Urheberrecht nur, wenn sie mit dem Verlag vorher vereinbart wurden. Im Einzelfall muß über die Zahlung einer Gebühr für die Nutzung fremden geistigen Eigentums entschieden werden. Das gilt für die Vervielfältigung durch alle Verfahren einschließlich Speicherung und jede Übertragung auf Papier, Transparente, Filme, Bänder, Platten und andere Medien.

Satz: Friedr. Vieweg \& Sohn, Braunschweig

Umschlaggestaltung: Peter Kohlhase, Lübeck 


\section{Vorwort}

Dieses Buch wendet sich an Studenten der Mathematik bis zum Vordiplom, an Informatiker und an Interessenten aus anderen naturwissenschaftlichen Fächern. Vorausgesetzt werden nur Grundkenntnisse der Analysis und der linearen Algebra.

Das Buch entstand aus einer einsemestrigen, dreistündigen Vorlesung, die der erstgenannte Verfasser wiederholt an der TU Braunschweig hielt. Es bringt eine elementare Einführung in die Methoden der numerischen Mathematik. Hauptanliegen ist es, die Grundideen der algorithmischen Lösung verschiedenster mathematischer Aufgaben möglichst klar werden zu lassen, um den Studenten in die Lage zu versetzen, verwandte Fragestellungen selbständig zu bearbeiten sowie die erlernten Prinzipien auf neue Probleme anzuwenden.

Um bei den Studenten die Freude an der Praxis zu wecken, sollte der Stoff möglichst lebendig dargestellt werden, ohne die Grundgedanken der Verfahren mit bezeichnungs- und beweistechnischen Schwierigkeiten zu überdecken.

Um praxisnah zu sein, sind die Algorithmen stets in einer Algol 60 ähnlichen Schreibweise angegeben, die unmittelbar programmierbar ist. An einfachen Beispielen wird der Ablauf der Verfahren demonstriert.

Besonderen Dank verdient Frl. Dr. Ingrid Brückner, die die erste Vorlesungsmitschrift anfertigte und am Entstehen des Buches wesentlich beteiligt war.

Für ihre Mithilfe beim Lesen der Korrekturen danken wir Frl. Dr. Brückner, Herrn Prof. Dr. Homuth und Herrn cand. math. Jürgen Rüger. 


\section{Inhaltsverzeichnis}

Vorwort $\ldots \ldots \ldots \ldots \ldots \ldots \ldots \ldots \ldots \ldots \ldots \ldots \ldots \ldots \ldots \ldots \ldots$ III

\section{Grundbegriffe}

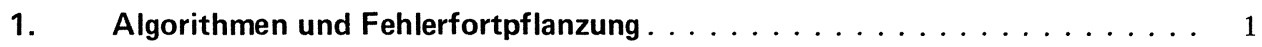

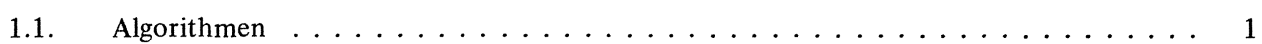

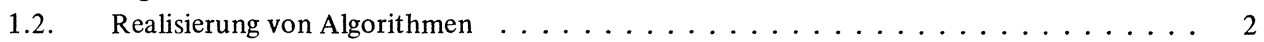

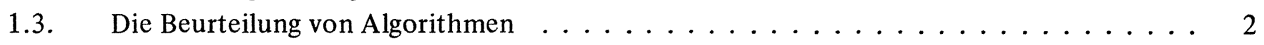

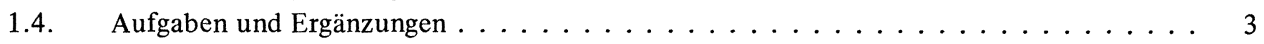

2. Matrizen ......................... 4

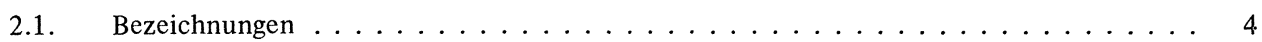

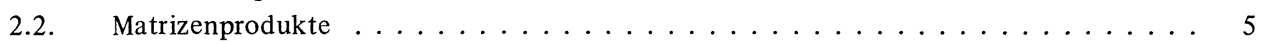

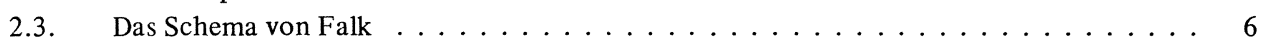

2.4. Rang und Determinante . . . . . . . . . . . . . . . . . . 7

2.5. Norm und Konvergenz . . . . . . . . . . . . . . . . . . 8

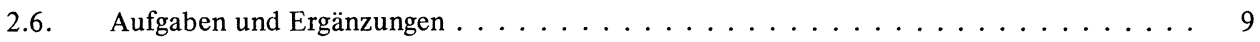

\section{Lineare Gleichungen und Ungleichungen}

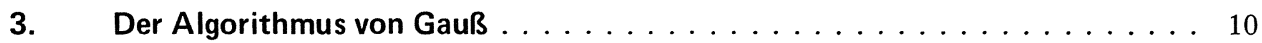

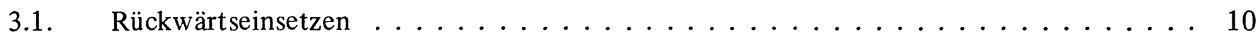

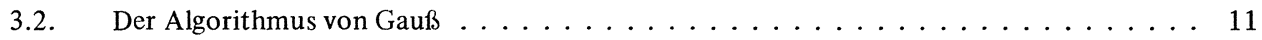

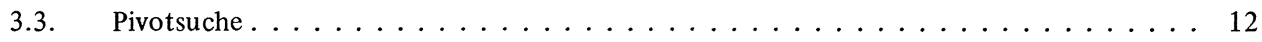

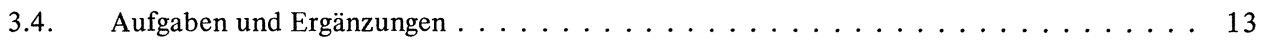

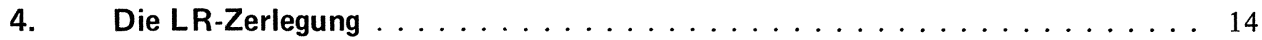

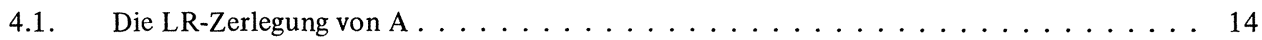

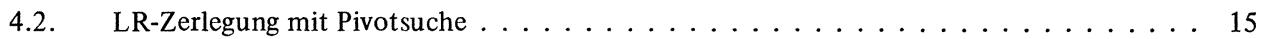

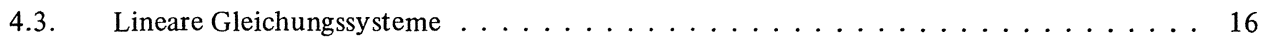

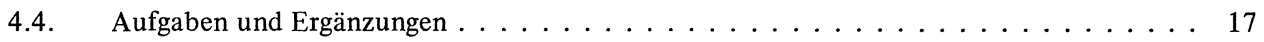

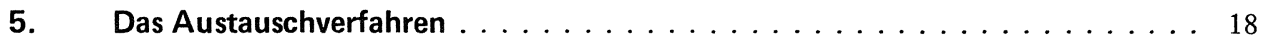

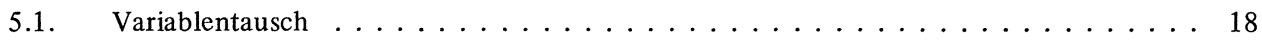

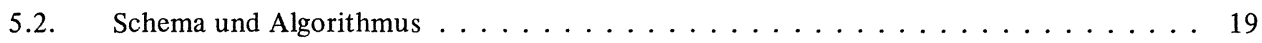

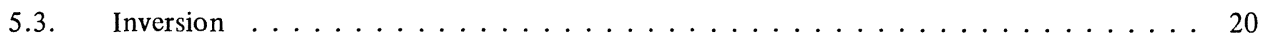

5.4. Lineare Gleichungen . . . . . . . . . . . . . . . . . . . 21

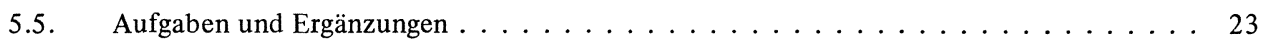




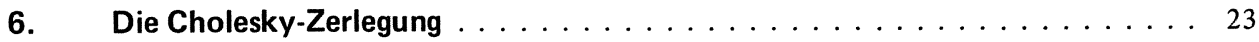

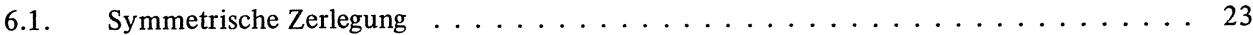

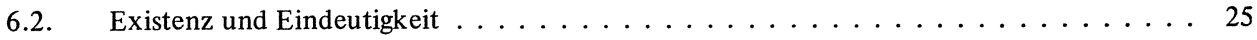

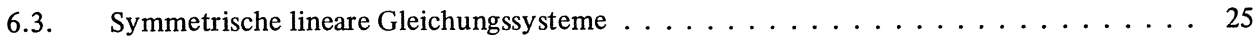

6.4. Nachiteration . . . . . . . . . . . . . . . . . . . . 26

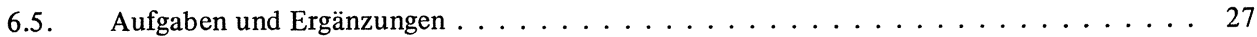

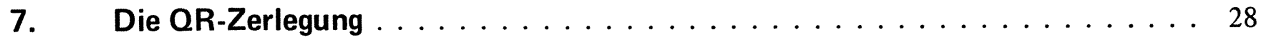

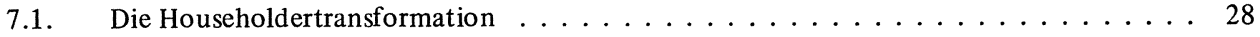

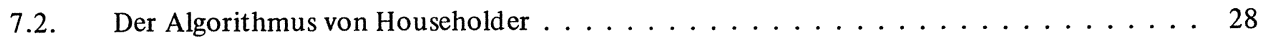

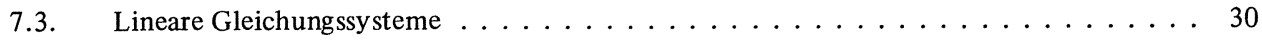

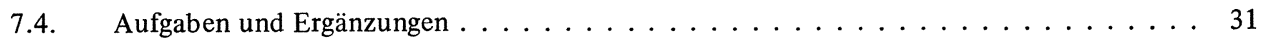

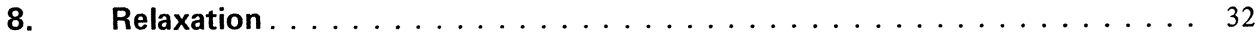

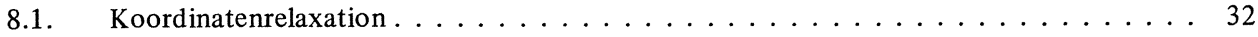

8.2. Konvergenz bei diagonaldominanten Matrizen $\ldots \ldots \ldots \ldots \ldots \ldots \ldots \ldots \ldots$

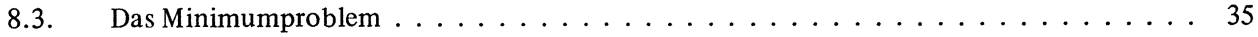

8.4. Konvergenz bei symmetrischen, positiv definiten Matrizen . . . . . . . . . . . 37

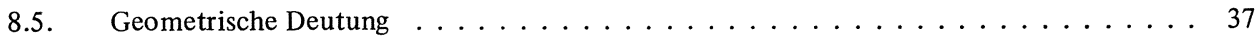

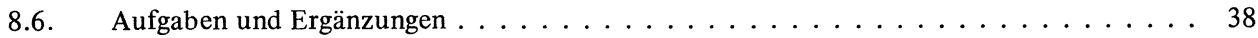

9. Lineares Ausgleichen . . . . . . . . . . . . . . . . . . 39

9.1. Überbestimmte lineare Gleichungssysteme . . . . . . . . . . . . . . . 39

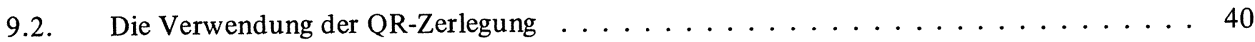

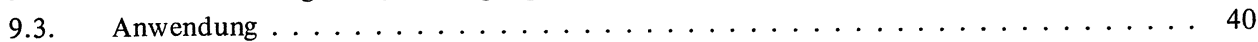

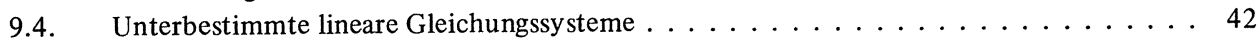

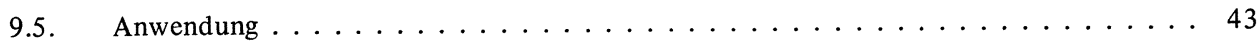

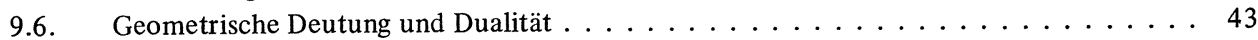

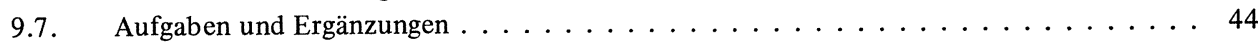

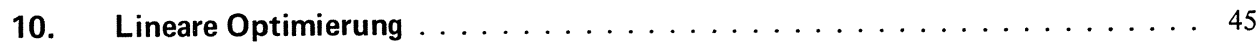

10.1. Lineare Ungleichungen und lineares Programm . . . . . . . . . . . 45

10.2. Eckentausch und Simplexverfahren $\ldots \ldots \ldots \ldots \ldots \ldots \ldots \ldots \ldots$

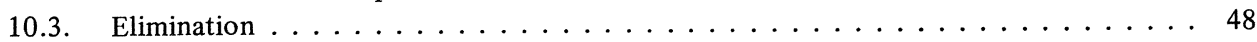

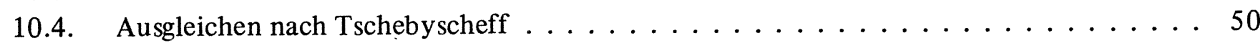

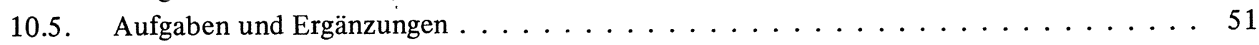

\section{Iteration}

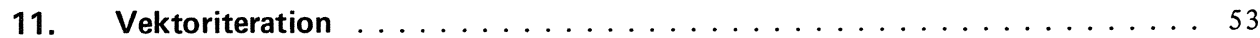

11.1. Das Eigenwertproblem für Matrizen $\ldots \ldots \ldots \ldots \ldots \ldots \ldots \ldots \ldots \ldots \ldots$

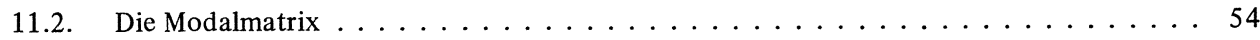

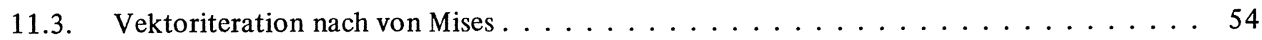

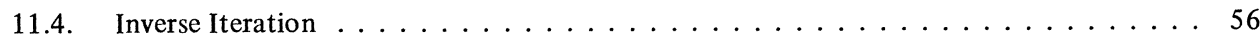

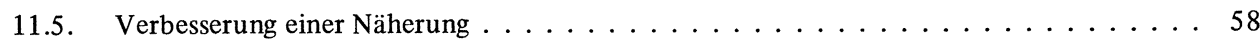

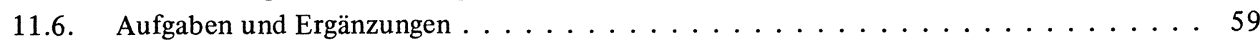




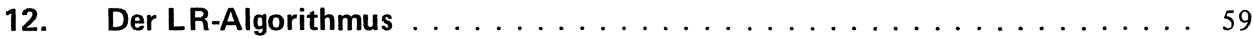

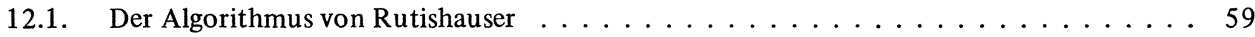

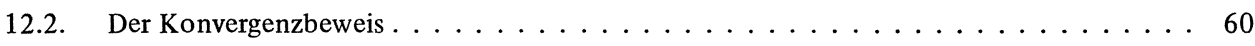

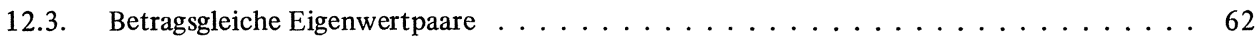

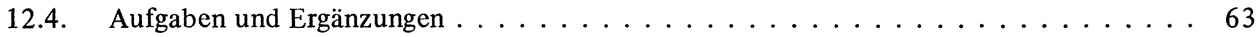

13. Eindimensionale Iteration $\ldots \ldots \ldots \ldots \ldots \ldots \ldots \ldots \ldots \ldots$

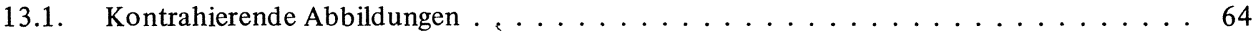

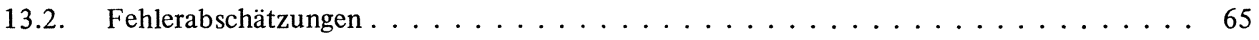

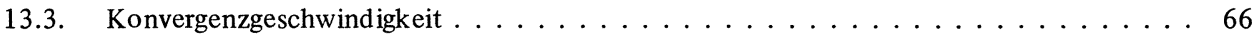

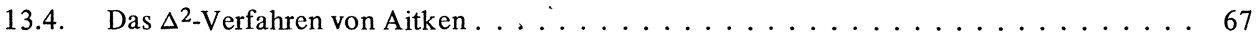

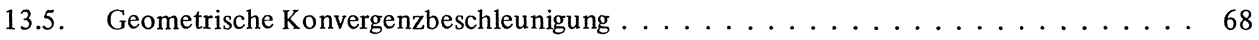

13.6. Nullstellen . . . . . . . . . . . . . . . . . . . . . . . . . 69

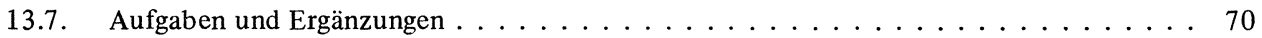

14. Mehrdimensionale Iteration $\ldots \ldots \ldots \ldots \ldots \ldots \ldots \ldots \ldots$

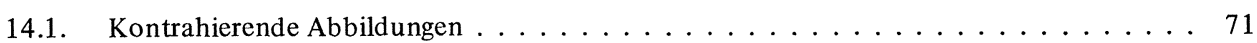

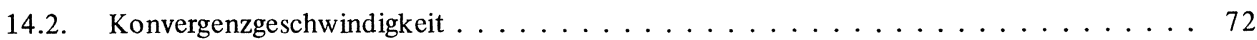

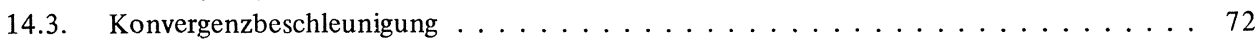

14.4. Nullstellen von Systemen . . . . . . . . . . . . . . . . . . . . . 73

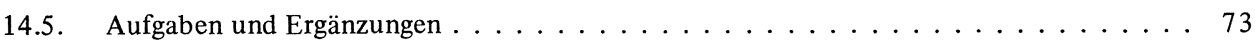

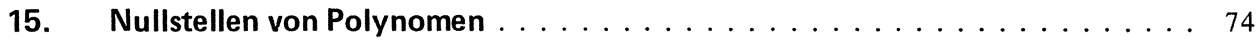

15.1. Das Horner-Schema . . . . . . . . . . . . . . . . . . . . . . . . . . 74

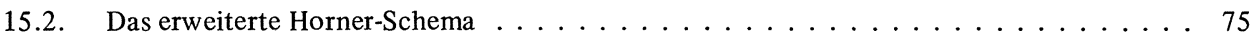

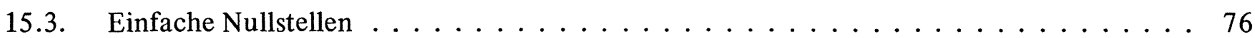

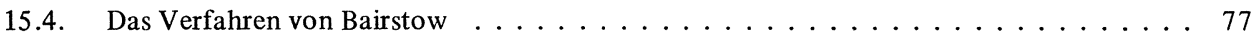

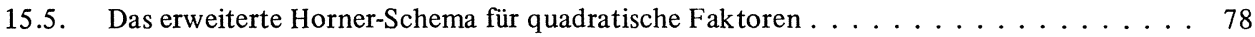

15.6. Aufgaben und Ergänzungen . . . . . . . . . . . . . . . . . . . . . . 79

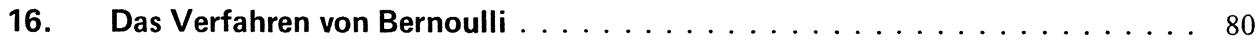

16.1. Lineare Differenzengleichungen $\ldots \ldots \ldots \ldots \ldots \ldots \ldots \ldots$

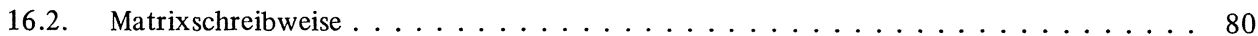

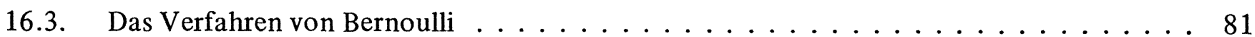

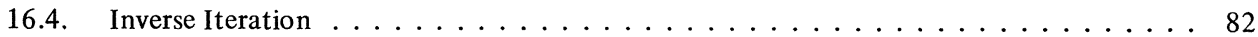

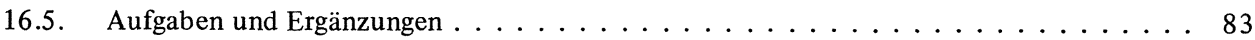

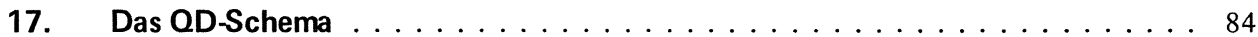

17.1. Der LR-Algorithmus für tridiagonale Matrizen $\ldots \ldots \ldots \ldots \ldots \ldots \ldots \ldots$

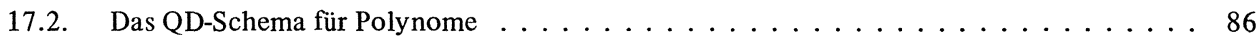

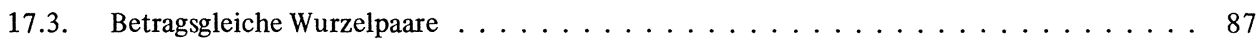

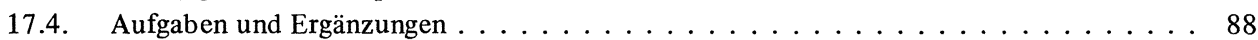




\section{Interpolation und diskrete Approximation}

18. Interpolation . . . . . . . . . . . . . . . . . . . 89

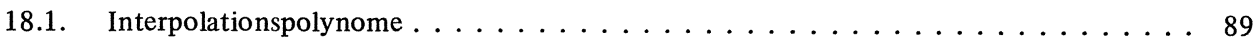

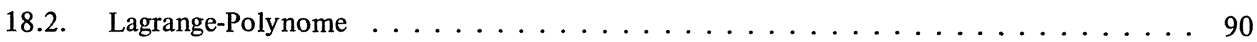

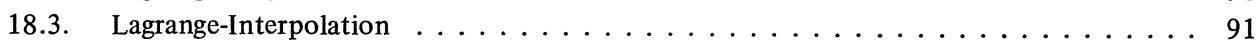

18.4. Newton-Interpolation . . . . . . . . . . . . . . . . . . 93

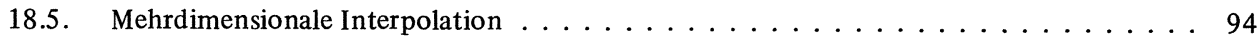

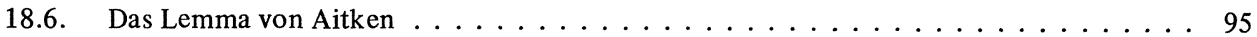

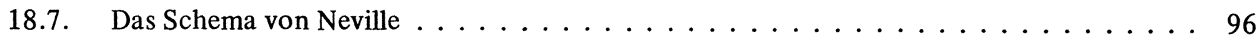

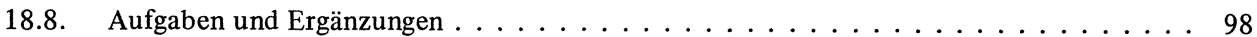

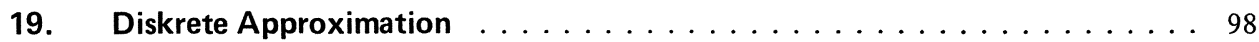

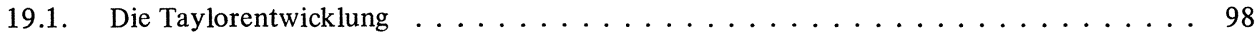

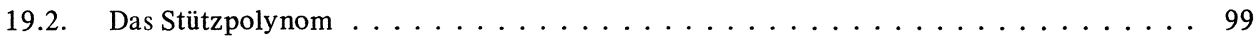

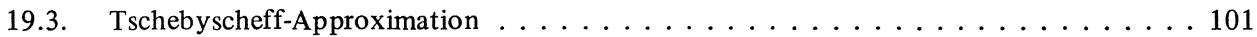

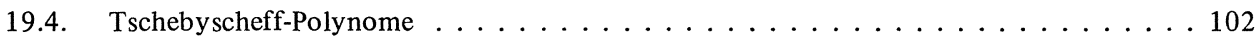

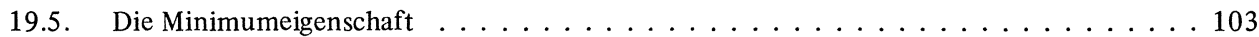

19.6. Entwicklung nach Tschebyscheff-Polynomen . . . . . . . . . . . . . . . . 104

19.7. Das Ökonomisieren eines Polynoms . . . . . . . . . . . . . . . . . . . . 105

19.8. Die Methode der kleinsten Quadrate . . . . . . . . . . . . . . . . . . . 105

19.9. Die Orthogonalität der Tschebyscheff-Polynome . . . . . . . . . . . . . . . 106

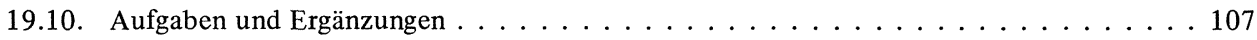

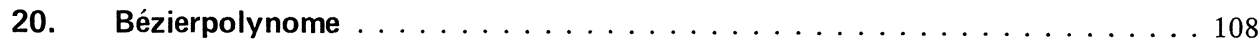

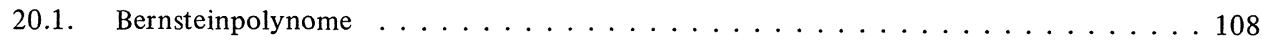

20.2. Bézier-Polynome . . . . . . . . . . . . . . . . . . . . 109

20.3. Die Konstruktion von Punkt und Tangente . . . . . . . . . . . . . . . . 110

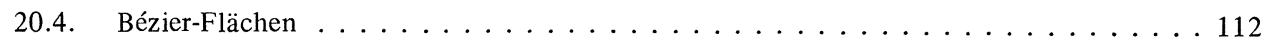

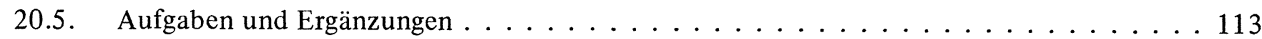

21. Splines und Subsplines . . . . . . . . . . . . . . . . . . . . . 114

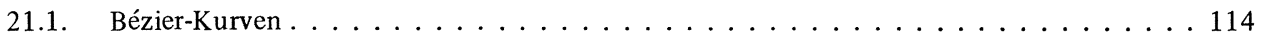

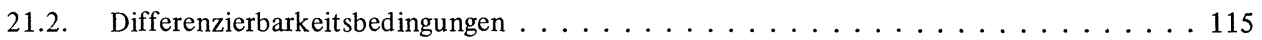

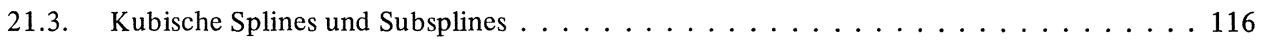

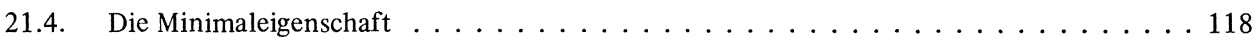

21.5. Aufgaben und Ergänzungen . . . . . . . . . . . . . . . . . . . . . 119

\section{Numerische Differentiation und Integration}

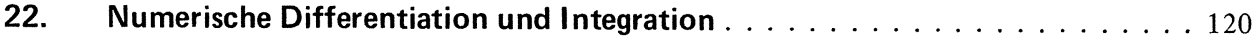

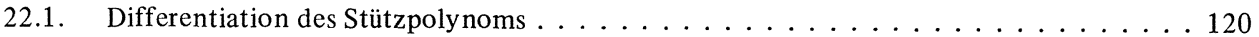

22.2. Fehlerabschätzung für die numerische Differentiation $\ldots \ldots \ldots \ldots \ldots \ldots \ldots \ldots \ldots$

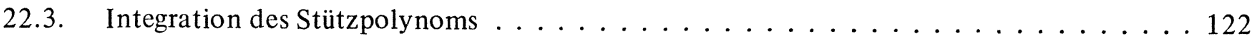

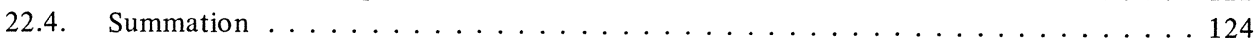

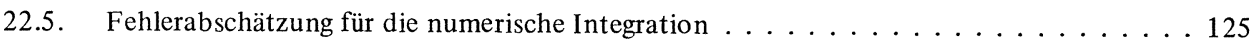

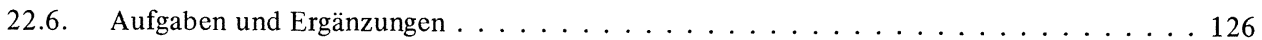




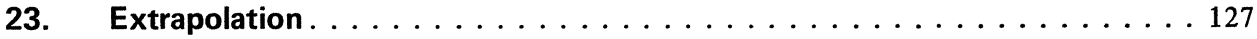

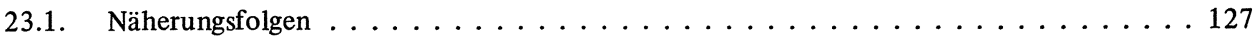

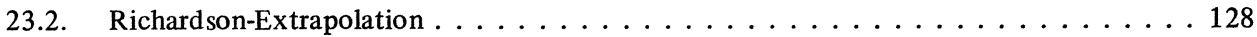

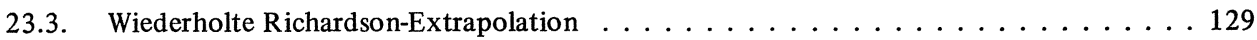

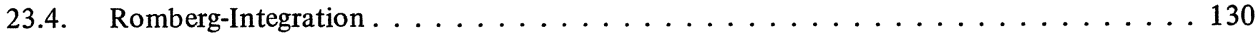

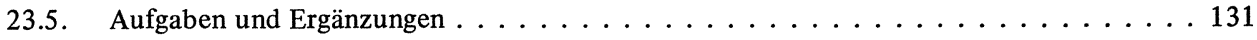

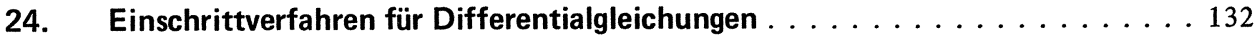

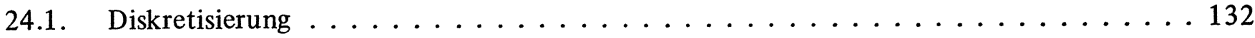

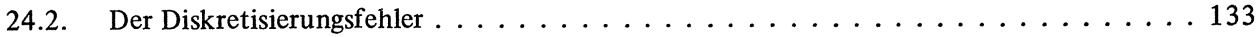

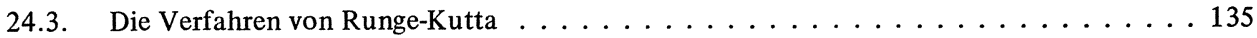

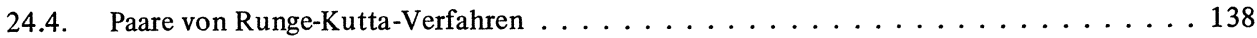

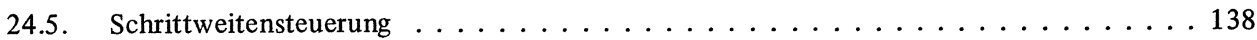

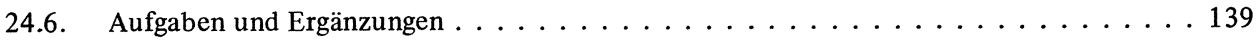

25. Lineare Mehrschrittverfahren für Differentialgleichungen $\ldots \ldots \ldots \ldots \ldots 141$

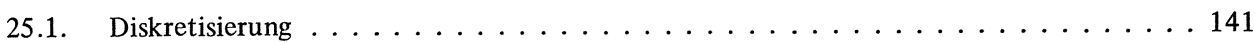

25.2. Die Konvergenz eines Mehrschrittverfahrens . . . . . . . . . . . . . . . . 143

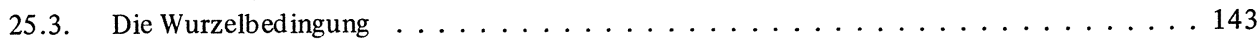

25.4. Hinreichende Konvergenzbedingung . . . . . . . . . . . . . . . . . . . . . . 144

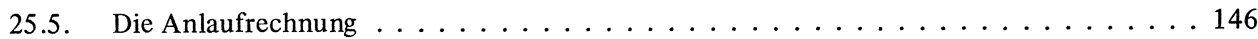

25.6. Prediktor-Korrektor Verfahren . . . . . . . . . . . . . . . . . . . . . 147

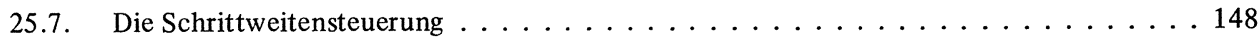

25.8. Vergleich von Einschritt- und Mehrschrittverfahren . . . . . . . . . . . . . . . 149

25.9. Aufgaben und Ergänzungen . . . . . . . . . . . . . . . . . . . . . . . 149

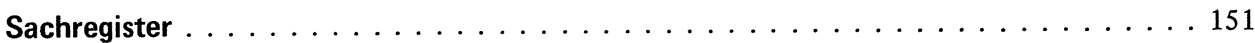

